\title{
Design of a Preview Controller for Discrete-Time Systems Based on LMI
}

\author{
Li Li and Fucheng Liao \\ School of Mathematics and Physics, University of Science and Technology Beijing, Beijing 100083, China \\ Correspondence should be addressed to Fucheng Liao; fcliao@ustb.edu.cn
}

Received 26 September 2015; Revised 2 December 2015; Accepted 10 December 2015

Academic Editor: Luis J. Yebra

Copyright ( 92015 L. Li and F. Liao. This is an open access article distributed under the Creative Commons Attribution License, which permits unrestricted use, distribution, and reproduction in any medium, provided the original work is properly cited.

\begin{abstract}
A preview controller design method for discrete-time systems based on LMI is proposed. First, we use the difference between a system state and its steady-state value, instead of the usual difference between system states, to transform the tracking problem into a regulator problem. Then, based on the Lyapunov stability theory and linear matrix inequality (LMI) approach, the preview controller ensuring asymptotic stability of the closed-loop system for the derived augmented error system is found. And an extended functional observer is designed in this paper which can achieve disturbance attenuation in the estimation process; as a result, the state of the system can be reconstructed rapidly and accurately. The controller gain matrix is obtained by solving an LMI problem. By incorporating the controller obtained into the original system, we obtain the preview controller of the system under consideration. To make sure that the output tracks the reference signal without steady-state error, an integrator is introduced. The numerical simulation example also illustrates the effectiveness of the results in the paper.
\end{abstract}

\section{Introduction}

The research problem of preview control theory is as follows: when the reference signal or exogenous disturbance is known or can be previewable, how can we take full advantage of the known future reference signal or disturbance signal to improve the tracking performance of a closed-loop system? In many cases, the future target signal or external disturbance for servo systems is known. For example, in vehicle active suspension [1,2], wind turbines [3], the robot system [4], and so on, their destination paths are specified in advance. We naturally seek to ascertain how to take full advantage of the known future information to improve the control performance or tracking performances. When considering these applications, preview control theory mentioned here will be used.

Preview control has been around since the 1960s and has been the subject of extensive, in-depth research. Reference [5] discussed the optimal preview control problem for multirate discrete-time systems subject to polynomial previewable desired output and polynomial previewable disturbance signals. Reference [6] studied the optimal preview control for a class of time-varying discrete systems, ingeniously overcoming the difficulty that the differential operator is nonlinear.
Using some techniques, [7] combined preview control theory with linear discrete-time descriptor systems to give the preview controller design method. Using $\mathrm{H}_{\infty}$ and $\mathrm{H}_{2}$ criteria, [812] converted the tracking problems of the original systems into standard $H_{\infty}$ and $H_{2}$ control problems by using certain techniques. And the design of preview controllers was given using existing theories. References [13, 14] presented the methods to design preview controllers based on Min-Max control in game theory.

Linear matrix inequality (LMI) is an effective method for robust control theory and has been applied to all aspects of systems and control [15]. The preview control problem for a class of discrete-time systems is presented in this paper. Combining the method of preview control theory with the LMI technique, a preview controller design is proposed. The proposed controller guarantees that the output of the closed-loop system can accurately track the reference signal. This paper adopts the method in [16] to construct an augmented error system, and the sufficient condition of asymptotic stability of the closed-loop system is derived using the second method of Lyapunov. References [17-19] considered the preview control for a polytopic uncertain system by LMI. However, they still adopted the difference method in preview control theory 
to construct an augmented error system; as a result, this constructive method cannot be extended to general uncertain systems. The paper uses the state transformation method instead of the classical difference method to construct the augmented error system and then considers the preview control problem by combining the second method of Lyapunov with the LMI technique. The benefit is that the results in this paper can easily be extended to uncertain systems.

Notations. $R^{n}$ and $R^{n \times n}$ denote the $n$-dimensional real vector space and $n \times n$ matrix space, respectively. $P>0(P \geq 0)$ denotes the notion that matrix $P$ is positive definite (positive semidefinite). $P>Q(P \geq Q)$ denotes $P-Q>0(P-Q \geq 0)$. $A$ is stable; that is, the absolute values of the eigenvalues of $A$ are strictly less than 1; $I$ denotes the identity matrix, and its dimension can be known from the context of the narrative.

\section{Problem Formulation and Basic Assumptions}

Consider the discrete-time system

$$
\begin{aligned}
x(k+1) & =A x(k)+B u(k)+E w(k), \\
y(k) & =C x(k),
\end{aligned}
$$

where $x(k) \in R^{n}$ is the state vector, $u(t) \in R^{m}$ is the control input vector, $y(k) \in R^{q}$ is the controlled output vector, $w(k) \in$ $R^{l}$ is the disturbance vector and $w(k) \in l_{2}$, and $A, B, C$, and $E$ are known real constant matrices with appropriate dimensions.

Let $r(k) \in R^{q}$ be the reference signal and define the error signal as

$$
e(k)=y(k)-r(k)
$$

In this paper, our objective is to design a preview controller in such a manner that the output $y(k)$ of the closedloop system tracks the reference signal $r(k)$ even in the presence of exogenous disturbances; that is,

$$
\lim _{k \rightarrow \infty} e(k)=\lim _{k \rightarrow \infty}(y(k)-r(k))=0 .
$$

Basic assumptions are as follows.

(A1) $\operatorname{rank}\left[\begin{array}{cc}A-I & B \\ C & 0\end{array}\right]=n+q$ (full-row rank).

(A1) is the standard assumption of the servomechanism design problems. It can imply that the system $(C, A, B)$ has no transmission zeros at $z=1$.

We assume that the reference signal and the exogenous disturbance are previewable, as more specifically described below.

(A2) Assume the preview length of the reference signal $r(k)$ is $M_{R}$; that is, at each time $k, M_{R}$ future values of $r(k+$ $1), r(k+2), \ldots, r\left(k+M_{R}\right)$ as well as the present and past values of the reference signal are available. The future values of the reference signal beyond $k+M_{R}$ are constant vector $r$ :

$$
r(k+j)=r, \quad j=M_{R}+1, M_{R}+2, M_{R}+3, \ldots
$$

We assume that the reference signal $r(k)$ converges to constant vector $r$ as time $k$ goes to infinity. That is,

$$
\lim _{k \rightarrow \infty} r(k)=r .
$$

(A3) Assume the preview length of the exogenous disturbance $w(k)$ is $M_{d}$; that is, at each time $k, M_{d}$ future values of $w(k+1), w(k+2), \ldots, w\left(k+M_{d}\right)$ as well as the present and past values of the exogenous disturbance are available. The future values of the exogenous disturbance beyond $k+M_{d}$ are zero:

$$
w(k+i)=0, \quad i=M_{d}+1, M_{d}+2, M_{d}+3, \ldots
$$

Remark 1. (A2) and (A3) are standard assumptions for preview control theory. The latter parts of (A2) and (A3) are based on the fact that, according to the characteristic of the control system itself, the previewable signals have a significant effect on the performance of the control system only for a certain time period; therefore, we can ignore the reference signal and the exogenous disturbance when they exceed the preview length. Here, we assume that the reference signal and the exogenous disturbance when they exceed the preview length are any constants. In fact, a regular feedback control system does not take full advantage of the known future reference signal and the exogenous disturbance, or equivalently, the preview length is zero.

The Schur complement lemma will be used in this paper. Lemma 2 (see [20]). Consider matrix $S=\left[\begin{array}{ll}S_{11} & S_{12} \\ S_{12}^{T} & S_{22}\end{array}\right]$, where $S_{11}$ and $S_{22}$ are symmetric matrices and invertible; then the following three conditions are equivalent:

(i) $S<0$.

(ii) $S_{11}<0, S_{22}-S_{12}^{T} S_{11}^{-1} S_{12}<0$.

(iii) $S_{22}<0, S_{11}-S_{12} S_{22}^{-1} S_{12}^{T}<0$.

To save space, we directly give the following lemma for stability analysis of the system by Lyapunov stability theory.

Lemma 3 (see [21]). The system $x(k+1)=A x(k)$ is asymptotically stable if and only if there is a positive definite matrix $P$ such that

$$
A^{T} P A-P<0 .
$$

\section{Derivation of the Augmented Error System}

We derive an augmented error system to transform the tracking problem into a regulator problem and consider the LMI technique to design a preview controller.

If the controlled output $y(k)$ of the closed-loop system for system (1) can track the reference signal $r(k)$, there exist constant vectors $x(\infty)=x^{*}$ and $u(\infty)=u^{*}$ satisfying the equation of system (1). On both sides of the system equation and observation equation, letting $k$ go to infinity, we obtain

$$
\begin{gathered}
x^{*}=A x^{*}+B u^{*}, \\
r=C x^{*} .
\end{gathered}
$$


That is,

$$
\left[\begin{array}{cc}
A-I & B \\
C & 0
\end{array}\right]\left[\begin{array}{l}
x^{*} \\
u^{*}
\end{array}\right]=\left[\begin{array}{l}
0 \\
I
\end{array}\right] r .
$$

By (A1), the coefficient matrix of the equation with $x^{*}$ and $u^{*}$ and the augmented matrix have the same rank; thus, at least one solution exists. Without loss of generality, we take a fixed solution which is still represented by $x^{*}$ and $u^{*}$.

It should be noted that (A1) implies $m \geq q$; that is, the number of control variables must be greater than or equal to that of the output variables to be controlled. This is quite common in practical control problems. And when $m=q, x^{*}$ and $u^{*}$ are existing and unique.

Define new vectors

$$
\begin{aligned}
& \tilde{x}(k)=x(k)-x^{*}, \\
& \tilde{u}(k)=u(k)-u^{*}, \\
& \tilde{y}(k)=y(k)-y^{*}, \\
& \tilde{r}(k)=r(k)-r .
\end{aligned}
$$

Combining (1) and (9) gives

$$
\begin{aligned}
\tilde{x}(k+1) & =A \tilde{x}(k)+B \tilde{u}(k)+E w(k), \\
\tilde{y}(k) & =C \tilde{x}(k) .
\end{aligned}
$$

And from (2), we obtain

$$
e(k)=C \tilde{x}(k)-\tilde{r}(k) .
$$

According to (A2), $\widetilde{r}(k)$ satisfies the following properties: at each time $k, \widetilde{r}(k), \widetilde{r}(k+1), \widetilde{r}(k+2), \ldots, \widetilde{r}\left(k+M_{R}\right)$ are known and

$$
\tilde{r}(k+j)=0, \quad j=M_{R}+1, M_{R}+2, M_{R}+3, \ldots .
$$
below.

The derivation of the augmented error system is given

First of all, combining (11) and (12) gives

$$
\begin{aligned}
e(k+1) & =C \tilde{x}(k+1)-\tilde{r}(k+1) \\
& =C[A \tilde{x}(k)+B \tilde{u}(k)+E w(k)]-\tilde{r}(k+1) .
\end{aligned}
$$

Then, constructing vectors

$$
\begin{gathered}
X_{R}(k)=\left[\begin{array}{c}
\tilde{r}(k) \\
\tilde{r}(k+1) \\
\vdots \\
\tilde{r}\left(k+M_{R}\right)
\end{array}\right], \\
X_{W}(k)=\left[\begin{array}{c}
w(k) \\
w(k+1) \\
\vdots \\
\vdots \\
w\left(k+M_{d}\right)
\end{array}\right]
\end{gathered}
$$

and matrices

$$
\begin{aligned}
A_{R} & =\left[\begin{array}{ccccc}
0 & I & & & 0 \\
0 & \ddots & \ddots & & \\
\vdots & & \ddots & \ddots & \\
0 & \cdots & \cdots & 0 & I \\
0 & \cdots & \cdots & 0 & 0
\end{array}\right], \\
A_{W} & =\left[\begin{array}{lllll}
0 & I & & & 0 \\
0 & \ddots & \ddots & & \\
\vdots & & \ddots & \ddots & \\
0 & \cdots & \cdots & 0 & I \\
0 & \cdots & \cdots & 0 & 0
\end{array}\right],
\end{aligned}
$$

it follows from (A2) and (A3) that we can obtain the equations as follows:

$$
\begin{gathered}
X_{R}(k+1)=A_{R} X_{R}(k), \\
X_{W}(k+1)=A_{W} X_{W}(k),
\end{gathered}
$$

where $X_{R}(k) \in R^{\left(M_{R}+1\right) q}, X_{W}(k) \in R^{\left(M_{d}+1\right) l}, A_{R} \in$ $R^{\left[\left(M_{R}+1\right) q\right] \times\left[\left(M_{R}+1\right) q\right]}$, and $A_{W} \in R^{\left[\left(M_{d}+1\right) l\right] \times\left[\left(M_{d}+1\right) l\right]}$. By (11), (14), and (17), we obtain the formal system

$$
\widehat{x}(k+1)=\widehat{A} \widehat{x}(k)+\widehat{B} \widetilde{u}(k),
$$

where

$$
\begin{aligned}
\widehat{x}(k) & =\left[\begin{array}{c}
e(k) \\
\tilde{x}(k) \\
X_{R}(k) \\
X_{W}(k)
\end{array}\right], \\
\widehat{A} & =\left[\begin{array}{llll}
0 & C A & G_{P R} & G_{P W} \\
0 & A & 0 & G_{W} \\
0 & 0 & A_{R} & 0 \\
0 & 0 & 0 & A_{W}
\end{array}\right], \\
\widehat{B} & =\left[\begin{array}{cc}
C B \\
B \\
0 &
\end{array}\right], \\
G_{P R} & =\left[\begin{array}{llll}
0 & -I & \cdots & 0
\end{array}\right], \\
G_{W} & =\left[\begin{array}{llll}
E & 0 & \cdots & 0
\end{array}\right], \\
G_{P W} & =\left[\begin{array}{llll}
C E & 0 & \cdots & 0
\end{array}\right]=C G_{W} .
\end{aligned}
$$

Note that the main characteristics of the formal system (18) are that both $X_{R}(k)$ and $X_{W}(k)$ with future information 
are a part of the state vector. Thus, system (18) contains the future information of the reference signal and disturbance signal. It should be pointed out that $\tilde{u}(k)$ replaces the difference of $\widetilde{u}(k)$ in the formal system (18); as a result, the controller obtained of system (18) by the LMI approach does not include the integral of error $e(k)$; therefore, the final closed-loop system does not contain an integrator that helps to eliminate the static error. For this, we adopt the method in [22] to introduce the discrete integrator defined by

$$
v(k+1)=v(k)+e(k) .
$$

Namely,

$$
v(k)=\sum_{j=0}^{k-1} e(j)+v(0),
$$

where $v(0)$ can be assigned as needed.

Define $X(k)=\left[\begin{array}{l}\widehat{x}(k) \\ v(k)\end{array}\right]$ again, and combining (18) and (20), we obtain

$$
X(k+1)=F X(k)+G \tilde{u}(k)
$$

where

$$
\begin{aligned}
& F=\left[\begin{array}{cccc|c}
0 & C A & G_{P R} & G_{P W} & 0 \\
0 & A & 0 & G_{W} & 0 \\
0 & 0 & A_{R} & 0 & 0 \\
0 & 0 & 0 & A_{W} & 0 \\
\hline I & 0 & 0 & 0 & I
\end{array}\right]=\left[\begin{array}{cc}
\widehat{A} & 0 \\
C_{E} & I
\end{array}\right], \\
& G=\left[\begin{array}{c}
C B \\
B \\
0 \\
0 \\
0
\end{array}\right]=\left[\begin{array}{c}
\widehat{B} \\
0
\end{array}\right] .
\end{aligned}
$$

System (22) is the derived augmented error system. Because $e(k)$ is a part of the vector $X(k)$, if we can design a state feedback

$$
\widetilde{u}(k)=K X(k)
$$

to make the closed-loop system of system (22) asymptotically stable and we have $\lim _{k \rightarrow \infty} X(k)=0$, then one gets $\lim _{k \rightarrow \infty} e(k)=0$. Thus, the state feedback is applied to system (1) and the output $y(k)$ of the corresponding closed-loop system tracks the reference signal $r(k)$ without steady-state error.

\section{Design of a Preview Controller}

As mentioned above, the purpose is accomplished as long as the state feedback obtained of system (22) is in the form as (24) and it makes the closed-loop system of system (22) asymptotically stable. We will give the controller gain matrix in (24) by using the related theory and LMI methods.
Note that when the control input is determined by (24), we see that the closed-loop system of system (22) is given by

$$
X(k+1)=(F+G K) X(k) .
$$

Below is the first important theorem in this paper.

Theorem 4. Suppose that (A1)-(A3) are satisfied. If there exist a positive definite symmetric matrix $M$ and a matrix $Y$ such that

$$
\left[\begin{array}{cc}
-M & M^{T} F^{T}+Y^{T} G^{T} \\
F M+G Y & -M
\end{array}\right]<0
$$

then the closed-loop system (25) is asymptotically stable, where the state feedback gain matrix $K=Y M^{-1}$, and the control law

$$
\tilde{u}(k)=Y M^{-1} X(k) .
$$

Proof. From Lemma 3, the closed-loop system (25) is asymptotically stable if and only if there is a positive definite matrix $P$ such that

$$
(F+G K)^{T} P(F+G K)-P<0 .
$$

Applying the Schur complement and $P>0,(28)$ is equivalent to

$$
\left[\begin{array}{cc}
-P & F^{T}+K^{T} G^{T} \\
F+G K & -P^{-1}
\end{array}\right]<0 .
$$

We perform the congruent transformation on the left side of (29): premultiplying an invertible symmetric matrix $\operatorname{diag}\left\{P^{-1} I\right\}$ and meanwhile postmultiplying the transposition of this matrix (namely, itself) and then denoting $M=$ $P^{-1}$ and $Y=K M$. It follows that we can obtain the condition of Theorem 4, namely, the matrix of the left side of (26). Since the congruent transformation does not change the positive definiteness, (26) is satisfied if and only if (29) is satisfied. From the above reasoning process, we obtain that (26) and (28) are equivalent. By Lemma 3, Theorem 4 holds.

Synthesis and derivations on Theorem 4 show that if LMI (26) has a feasible solution, there exists state feedback $\tilde{u}(k)=$ $K X(k)$ for system $(22)$ such that $(F+G K)$ is a stability matrix. In other words, the pair $(F, G)$ is stabilizable [23]. In the following, the $\mathrm{PBH}$ rank test [24] will be employed to prove the stabilizability of the augmented error system (22).

Lemma 5. The pair $(F, G)$ is stabilizable if and only if $(A, B)$ is stabilizable and $\left[\begin{array}{cc}A-I & B \\ C & 0\end{array}\right]$ has full-row rank.

Proof. By the PBH criteria [24], the pair $(F, G)$ is stabilizable if and only if the matrix

$$
\left[\begin{array}{ll}
F-s I & G
\end{array}\right]
$$

has full-row rank for any complex $s$ satisfying $|s| \geq 1$. 
Note that it follows from the structure of $A_{R}$ and $A_{W}$ that both $A_{R}-s I$ and $A_{W}-s I$ are nonsingular for any $s$ satisfying $|s| \geq 1$. From the expression of $F$ and $G$, we have

$$
\begin{aligned}
& {\left[\begin{array}{ll}
F-s I & G
\end{array}\right]} \\
& =\left[\begin{array}{ccccc|c}
-s I & C A & G_{P R} & G_{P W} & 0 & C B \\
0 & A-s I & 0 & G_{W} & 0 & B \\
0 & 0 & A_{R}-s I & 0 & 0 & 0 \\
0 & 0 & 0 & A_{W}-s I & 0 & 0 \\
I & 0 & 0 & 0 & (1-s) I & 0
\end{array}\right] .
\end{aligned}
$$

Elementary matrix operations do not change the rank of the matrix and $(1-s) I$ is nonsingular for any complex $s$ satisfying $|s| \geq 1$ and $s \neq 1$. By the elementary transformation of the matrix, we have

$$
\begin{aligned}
& {\left[\begin{array}{ll}
F-s I & G
\end{array}\right]} \\
& \longrightarrow\left[\begin{array}{ccccc|c}
-s I & 0 & 0 & 0 & 0 & 0 \\
0 & A-s I & 0 & 0 & 0 & B \\
0 & 0 & A_{R}-s I & 0 & 0 & 0 \\
0 & 0 & 0 & A_{W}-s I & 0 & 0 \\
0 & 0 & 0 & 0 & (1-s) I & 0
\end{array}\right] .
\end{aligned}
$$

Thus, the matrix $\left[\begin{array}{ll}F-s I & G\end{array}\right]$ is of full-row rank if and only if $\left[\begin{array}{ll}A-s I & B\end{array}\right]$ is of full-row rank.

$$
K=\left[\begin{array}{lllllllllll}
K_{e} & K_{x} & K_{R}(0) & K_{R}(1) & \cdots & K_{R}\left(M_{R}\right) & K_{d}(0) & K_{d}(1) & \cdots & K_{d}\left(M_{d}\right) & K_{v}
\end{array}\right] ;
$$

When $s=1$, we have from the elementary transformation of the matrix

$$
\begin{aligned}
{[F-s I} & G]_{s=1} \\
& \longrightarrow\left[\begin{array}{ccccc|c}
0 & C A & 0 & 0 & 0 & C B \\
0 & A-I & 0 & 0 & 0 & B \\
0 & 0 & A_{R}-I & 0 & 0 & 0 \\
0 & 0 & 0 & A_{W}-I & 0 & 0 \\
I & 0 & 0 & 0 & 0 & 0
\end{array}\right] \\
\longrightarrow & {\left[\begin{array}{ccccc|c}
0 & C & 0 & 0 & 0 & 0 \\
0 & A-I & 0 & 0 & 0 & B \\
0 & 0 & A_{R}-I & 0 & 0 & 0 \\
0 & 0 & 0 & A_{W}-I & 0 & 0 \\
I & 0 & 0 & 0 & 0 & 0
\end{array}\right] . }
\end{aligned}
$$

Thus, the matrix $\left[\begin{array}{ll}F-s I & G\end{array}\right]_{s=1}$ is of full-row rank if and only if $\left[\begin{array}{cc}A-I & B \\ C & 0\end{array}\right]$ is of full-row rank.

$$
\text { Lemma } 5 \text { holds. }
$$

Remark 6. If the condition of Theorem 4 is established, the pair $(A, B)$ is stabilizable and $\left[\begin{array}{cc}A-I & B \\ C & 0\end{array}\right]$ has full-row rank. And the stabilizability of $(A, B)$ and rank $\left[\begin{array}{cc}A-I & B \\ C & 0\end{array}\right]=n+q$ guarantee that system (1) is stabilizable via state feedback and has no zeros at $s=1$, and then these are necessary for the existence of a robustly stabilizing controller for the augmented error system (22). Therefore, the LMI approach is applied to preview control theory and the determination of a preview controller can be converted into a convex optimization problem in terms of linear matrix inequality; as a result, the design of the preview controller becomes a simple matter and some restricted conditions in [24] will be reduced.

We consider the control input of system (1).

When (A1)-(A3) are satisfied, the control input (24) of system (22) is obtained. We decompose the gain matrix $K$ then, (24) can be written as

$$
\begin{aligned}
u(k)= & u^{*}+K_{e} e(k)+K_{x} \tilde{x}(k)+\sum_{i=0}^{M_{R}} K_{R}(i) \tilde{r}(k+i) \\
& +\sum_{i=0}^{M_{d}} K_{d}(i) w(k+i)+K_{v} v(k)
\end{aligned}
$$

Substituting $\tilde{x}(k)=x(k)-x^{*}, \tilde{u}(k)=u(k)-u^{*}$, and $\tilde{r}(k)=$ $r(k)-r$ into the above equation, the following is obtained.
Theorem 7. Suppose that (A1)-(A3) are satisfied. The controller of system (1) can be taken as

$$
\begin{aligned}
u(k)= & K_{e} e(k)+K_{x} x(k)+\sum_{i=0}^{M_{R}} K_{R}(i)(r(k+i)-r) \\
& +\sum_{i=0}^{M_{d}} K_{d}(i) w(k+i)+K_{v}\left(\sum_{s=0}^{k-1} e(s)+v(0)\right) \\
& +\left[-K_{x} I\right]\left[\begin{array}{l}
x^{*} \\
u^{*}
\end{array}\right],
\end{aligned}
$$


where $K=Y M^{-1}, M>0$, and $Y$ can be determined by (26). Equation (34) determines the relationship between $K_{e}, K_{x}$, $K_{R}(0), \ldots, K_{R}\left(M_{R}\right), K_{d}(0), K_{d}(1), \ldots, K_{d}\left(M_{d}\right), K_{v}$, and $K$. Under the controller, the output $y(k)$ of the closed-loop system of system (1) tracks the reference signal $r(k)$ accurately.

We can see from the above equation that the preview controller of system (1) consists of six terms. The first term represents tracking error compensation, the second term represents the state feedback, the third term represents the feedforward or preview action based on the future information of the reference signal, the fourth term represents the feedforward or preview action based on the future information of the exogenous disturbance, the fifth term represents the integral action of the tracking error, and the sixth term represents the compensation by the initial and final values.

\section{Design of an Observer-Based Controller}

In practical application, the system state information of system (1) often cannot be obtained; thereby, the system state information of system (11) cannot be obtained either. Thus, we need to build an observer which reconstructs the state variables. If we use the method in [23] to design a state observer for system (1), then

$$
\widehat{x}(k+1)=A \widehat{x}(k)+B u(k)+L[y(k)-C \widehat{x}(k)] .
$$

Let the estimation error $e_{x}(k)=x(k)-\widehat{x}(k)$ and use (1) and (37) as follows:

$$
\begin{aligned}
e_{x}(k+1)= & x(k+1)-\widehat{x}(k+1) \\
= & A x(k)+B u(k)+E w(k)-A \widehat{x}(k) \\
& -B u(k)-L[y(k)-C \widehat{x}(k)] \\
= & (A-L C) e_{x}(k)+E w(k) .
\end{aligned}
$$

Obviously, the estimation error will be unavoidably affected by the disturbance $w(k)$; thus both the rate of convergence for estimating the state variables and the interference rejection will be affected [25-27]. To overcome this disadvantage, we design the extended functional observer to estimate the state $x(k)$ and the disturbance $w(k)$ simultaneously. Now, for simplicity of presentation, let $\bar{x}(k)=\left[\begin{array}{c}x(k) \\ w(k)\end{array}\right], \bar{E}=\left[\begin{array}{ll}I & 0\end{array}\right]$, and $\bar{A}=\left[\begin{array}{ll}A & E\end{array}\right]$; we have

$$
\bar{E} \bar{x}(k+1)=\bar{A} \bar{x}(k)+B u(k) .
$$

Now consider the observation equation of system (1) and the predictability of the disturbance; for the augmented system, we now ought to take the observation equation as

$$
y_{g}(k)=\bar{C} \bar{x}(k)
$$

where $\bar{C}=\left[\begin{array}{ll}{ }^{C} & \\ & \end{array}\right]$. Hence, we obtain

$$
\begin{aligned}
\bar{E} \bar{x}(k+1) & =\bar{A} \bar{x}(k)+B u(k), \\
y_{g}(k) & =\bar{C} \bar{x}(k) .
\end{aligned}
$$

Then, the state and the disturbance estimation problem can be converted into a problem of designing a state observer for the augmented system (41). Now for system (41) we consider the state observer given by

$$
\begin{aligned}
\eta(k+1) & =Z \eta(k)+H u(k)+P y_{g}(k), \\
\hat{\bar{x}}(k) & =\eta(k)+Q y_{g}(k),
\end{aligned}
$$

where $\widehat{\bar{x}}(k) \in R^{n+l}$ is the observer state, $\eta(k) \in R^{n+l}$ is an auxiliary variable, and $P, Q, Z$, and $H$ are constant matrices with appropriate dimensions that should be designed.

Note that $\operatorname{rank}[\overline{\bar{E}}]=\operatorname{rank}\left[\begin{array}{cc}I_{n} & 0 \\ C & 0 \\ 0 & I_{l}\end{array}\right]=n+l$ (full-column rank). It follows from the verification that

$$
\left[\begin{array}{ccc}
I_{n} & 0 & 0 \\
0 & 0 & I_{l}
\end{array}\right]\left[\begin{array}{ccc}
I_{n} & 0 & 0 \\
-C & I_{q} & 0 \\
0 & 0 & I_{l}
\end{array}\right]\left[\begin{array}{cc}
I_{n} & 0 \\
C & 0 \\
0 & I_{l}
\end{array}\right]=\left[\begin{array}{ll}
I_{n} & \\
& I_{l}
\end{array}\right]
$$

that is,

$$
\left[\begin{array}{ccc}
I_{n} & 0 & 0 \\
0 & 0 & I_{l}
\end{array}\right]\left[\begin{array}{ll}
I_{n} & 0 \\
C & 0 \\
0 & I_{l}
\end{array}\right]=\left[\begin{array}{c|cc}
I_{n} & 0 & 0 \\
0 & 0 & I_{l}
\end{array}\right]\left[\begin{array}{c}
\bar{E} \\
\bar{C}
\end{array}\right]=\left[\begin{array}{cc}
I_{n} & 0 \\
0 & I_{l}
\end{array}\right] .
$$

Then, we denote $M=\left[\begin{array}{c}I_{n} \\ 0\end{array}\right], N=\left[\begin{array}{ll}0 & 0 \\ 0 & I_{l}\end{array}\right]$ and then matrices $M$ and $N$ satisfy the equation

$$
M \bar{E}+N \bar{C}=I_{n+l} .
$$

Theorem 8. For the augmented system (41), the estimation error $e_{\bar{x}}(k)=\bar{x}(k)-\widehat{\bar{x}}(k)$ converges asymptotically to zero if there exists a matrix $\bar{L}$ such that $M \bar{A}-\bar{L} \bar{C}$ is stable. Furthermore, matrices $Z, H, P$, and $Q$ of the functional observer (42) can be determined as

$$
\begin{aligned}
& Z=M \bar{A}-L \bar{C}, \\
& H=M B \\
& P=\bar{L}+(M \bar{A}-\bar{L} \bar{C}) N, \\
& Q=N .
\end{aligned}
$$

Proof. Multiplying $M$ at both sides of the first formula for (41) yields

$$
M \bar{E} \bar{x}(k+1)=M \bar{A} \bar{x}(k)+M B u(k)
$$

and adding $N y_{g}(k+1)=N \bar{C} \bar{x}(k+1)$ to both sides of (47) yields

$$
\begin{aligned}
& (M \bar{E}+N \bar{C}) \bar{x}(k+1) \\
& \quad=M \bar{A} \bar{x}(k)+M B u(k)+N y_{g}(k+1) .
\end{aligned}
$$

Since (45) is $M \bar{E}+N \bar{C}=I_{n+l}$, (48) can be rewritten as

$$
\bar{x}(k+1)=M \bar{A} \bar{x}(k)+M B u(k)+N y_{g}(k+1) .
$$


Letting $d(k)=M \bar{B} u(k)+N y_{g}(k+1)$, system (49) can be rewritten as

$$
\begin{aligned}
\bar{x}(k+1) & =M \bar{A} \bar{x}(k)+d(k), \\
y_{g}(k) & =\bar{C} \bar{x}(k) .
\end{aligned}
$$

For system (50), we consider a state observer as follows:

$$
\begin{aligned}
\hat{\bar{x}}(k+1)= & M \bar{A} \hat{\bar{x}}(k)+d(k)+\bar{L}\left(y_{g}(k)-\bar{C} \hat{\bar{x}}(k)\right) \\
= & M \bar{A} \hat{\bar{x}}(k)+M B u(k)+N y_{g}(k+1) \\
& +\bar{L}\left(y_{g}(k)-\bar{C} \hat{\bar{x}}(k)\right) .
\end{aligned}
$$

To eliminate the term $y_{g}(k+1)$, we introduce an auxiliary variable $\eta(k)=\widehat{\bar{x}}(k)-N y_{g}(k)$, and the observer (51) turns into

$$
\begin{aligned}
\eta(k+1)= & (M \bar{A}-\bar{L} \bar{C}) \eta(k)+M B u(k) \\
& +(\bar{L}+(M \bar{A}-\bar{L} \bar{C}) N) y_{g}(k), \\
\widehat{\bar{x}}(k)= & \eta(k)+N y_{g}(k) .
\end{aligned}
$$

Comparing (42) with (52), parameter matrices of the functional observer (42) can be designed.

Subtracting (51) from (49) yields the following estimation error equation:

$$
e_{\bar{x}}(k+1)=(M \bar{A}-\bar{L} \bar{C}) e_{\bar{x}}(k) .
$$

Selecting a proper matrix $\bar{L}$ makes $(M \bar{A}-\bar{L} \bar{C})$ be stable; then there must be $\lim _{k \rightarrow \infty}[\bar{x}(k)-\hat{\bar{x}}(k)]=0$. Consequently, $x(k)$ can be reconstructed, and the estimates of state variables can be determined as $\widehat{x}(k)=\left[\begin{array}{ll}I_{n} & 0_{n \times l}\end{array}\right] \widehat{\bar{x}}(k)$.

Lemma 9. There exists the observer gain $\bar{L}$ such that $(M \bar{A}-$ $\overline{L C})$ is stable if and only if the pair $(C, A)$ is detectable.

Proof. If there exists a matrix $\bar{L}$ such that $(M \bar{A}-\bar{L} \bar{C})$ is stable, that is, $\left(\bar{A}^{T} M^{T}-\bar{C}^{T} \bar{L}^{T}\right)$ is stable, or, equivalently, the pair $\left(\bar{A}^{T} M^{T}, \bar{C}^{T}\right)$ is stabilizable, then we know the pair $(\bar{C}, M \bar{A})$ is detectable based on the duality principle. In the following, we will use $\mathrm{PBH}$ criteria to verify the condition of the detectability of the pair $(\bar{C}, M \bar{A})$.

By the PBH criteria [24], the pair $(\bar{C}, M \bar{A})$ is detectable if and only if the matrix

$$
\left[\begin{array}{c}
s I-M \bar{A} \\
\bar{C}
\end{array}\right]
$$

has full-column rank for any complex s satisfying $|s| \geq 1$. From the expression for $M \bar{A}$ and $\bar{C}$, we see that

$$
\left[\begin{array}{c}
s I-M \bar{A} \\
\bar{C}
\end{array}\right]=\left[\begin{array}{cc}
s I-A & -E \\
C A & (s+1) I+C E \\
\hline C & 0 \\
0 & I
\end{array}\right]
$$

$$
\longrightarrow\left[\begin{array}{cc}
s I-A & 0 \\
C A & 0 \\
\hline C & 0 \\
0 & I
\end{array}\right] \longrightarrow\left[\begin{array}{cc}
s I-A & 0 \\
s C & 0 \\
\hline C & 0 \\
0 & I
\end{array}\right]
$$

$$
\longrightarrow\left[\begin{array}{cr}
s I-A & 0 \\
0 & 0 \\
\hline C & 0 \\
0 & I
\end{array}\right] \text {. }
$$

The pair $(\bar{C}, M \bar{A})$ is detectable if and only if $(C, A)$ is detectable. Lemma 9 holds.

Remark 10. First we construct the augmented system (39) and reformulate the output equation. Then, the extended functional observer (42) is proposed for the formal system (41). By (53), an appropriate $\bar{L}$ is selected such that $(M \bar{A}-\bar{L} \bar{C})$ is stable $\left[M=\left[\begin{array}{c}I_{n} \\ 0\end{array}\right]\right.$ is a given matrix $]$, and the estimation error $e_{\bar{x}}(k)$ will converge asymptotically to zero.

Remark 11. $\bar{L}=\left[\begin{array}{cc}L_{1} & 0 \\ 0 & L_{2}\end{array}\right]$ is taken to be a quasi-diagonal matrix and then the coefficient matrix of system (53) is $M \bar{A}-\bar{L} \bar{C}=$ $\left[\begin{array}{cc}A-L_{1} C & E \\ 0 & -L_{2}\end{array}\right]$. System (53) can be rewritten as

$$
\left[\begin{array}{l}
e_{x}(k+1) \\
e_{w}(k+1)
\end{array}\right]=\left[\begin{array}{cc}
A-L_{1} C & E \\
0 & -L_{2}
\end{array}\right]\left[\begin{array}{l}
e_{x}(k) \\
e_{w}(k)
\end{array}\right],
$$

where $e_{x}(k)$ and $e_{w}(k)$ are the estimation errors of the system state and the disturbance, respectively. According to the estimation error equation (56), $L_{2}$ is taken to be stable. In fact, selecting $L_{2}$ can speed up the convergence rate for the disturbance estimation and reduce the interference for system state estimation. Thus, strong interference suppression can be realized. Furthermore, the issue of choosing $L_{1}$ making $A-L_{1} C$ stable has already been solved in linear system theory and is not attempted here.

Under the functional observer (42), we can derive the closed-loop system as follows:

$$
\begin{aligned}
& x(k+1)=A x(k)+B u(k)+E w(k), \\
& y(k)=C x(k), \\
& e(k)=y(k)-r(k), \\
& \eta(k+1) \\
& =(M \bar{A}-\bar{L} \bar{C}) \eta(k)+M B u(k) \\
& +(\bar{L}+(M \bar{A}-\bar{L} \bar{C}) N) y_{g}(k),
\end{aligned}
$$




$$
\begin{aligned}
\hat{\bar{x}}(k)= & \eta(k)+Q y_{g}(k), \\
u(k) & \\
= & u^{*}+K_{e} e(k)+K_{x}\left(\left[\begin{array}{ll}
I & 0
\end{array}\right] \widehat{\bar{x}}(k)-x^{*}\right) \\
& +\sum_{i=0}^{M_{R}} k_{R}(i)(r(k+i)-r) \\
& +\sum_{i=0}^{M_{d}} k_{d}(i) w(k+i)+K_{v}\left(\sum_{s=0}^{k-1} e(s)+v(0)\right) .
\end{aligned}
$$

$$
\begin{aligned}
& K=\left[\begin{array}{llll|ll}
-1.72182 & 4.10946 & 14.01371 & -0.00229 & 1.73271
\end{array}\right. \\
& K_{e}=-1.72182 \text {, } \\
& K_{x}=\left[\begin{array}{ll}
4.10946 & 14.01371
\end{array}\right] \text {, } \\
& K_{R}=\left[\begin{array}{lll}
-0.00229 & 1.73271 & 2.15569
\end{array}\right] \text {, } \\
& K_{d}=\left[\begin{array}{lll}
-0.90683 & -0.03124 & -0.01647
\end{array}\right] \text {, } \\
& K_{v}=-1.71040 \text {. }
\end{aligned}
$$

When $M_{R}=5, M_{d}=2$, we obtain

$K=\left[\begin{array}{lllll}K_{e} & K_{x} & K_{R} & K_{d} & K_{v}\end{array}\right] \in R^{1 \times 13}$,

$K_{e}=-1.95894$,

$K_{x}=\left[\begin{array}{ll}5.08080 & 15.11991\end{array}\right]$,

$K_{R}$

$=\left[\begin{array}{llllll}-0.02823 & 1.97058 & 2.34712 & 1.60436 & 0.97740 & 0.53439\end{array}\right]$,

$K_{d}=\left[\begin{array}{lll}-0.91174 & -0.03438 & -0.01831\end{array}\right]$,

$K_{v}=-1.89826$.

When $M_{R}=0, M_{d}=0$, we obtain

$$
\begin{aligned}
K & =\left[\begin{array}{lll}
K_{e} & K_{x} & K_{v}
\end{array}\right] \\
& =\left[\begin{array}{ll}
-1.75751 \mid 3.86489 & 13.55422 \mid-1.69170
\end{array}\right] .
\end{aligned}
$$

Let the initial conditions of $x(k)$ be $x(0)=\left[\begin{array}{l}0 \\ 0\end{array}\right]$ and let the initial conditions of $v(k)$ be $v(0)=3.45447, v(0)=3.58961$, and $v(0)=7.63391$, respectively. In addition, we take the exogenous disturbance as

$$
w(k)= \begin{cases}1.5, & 30 \leq k \leq 60 \\ 0, & \text { other. }\end{cases}
$$

Note that, from the simulation results, it can be seen that choosing $v(0)$ properly for different situations can eliminate the oscillation caused by $x^{*}$ and $u^{*}$.

\section{Numerical Example}

In system (1), let $A=\left[\begin{array}{cc}-0.7 & 1.2 \\ 1.2 & 0.4\end{array}\right], B=\left[\begin{array}{c}-0.23 \\ 0.12\end{array}\right], C=$ $\left[\begin{array}{ll}-0.65 & -1.2\end{array}\right]$, and $E=\left[\begin{array}{c}-0.2 \\ 0.1\end{array}\right]$. Through verification, $\left[\begin{array}{cc}A-I & B \\ C & 0\end{array}\right]$ satisfies full-row rank, and $(C, A)$ is detectable. Therefore, the system satisfies the basic assumptions.

We perform simulations for three situations; that is, the preview lengths of the tracking signal and the exogenous disturbance are $M_{R}=2, M_{d}=2, M_{R}=5, M_{d}=2$, and no preview (namely, $M_{R}=M_{d}=0$ ). According to Theorem 4, we use the LMI toolbox of MATLAB to determine matrix variables $M$ and $Y$ in LMI (26); then the state feedback gain matrix $K=Y M^{-1}$ is obtained naturally.

When $M_{R}=2, M_{d}=2$, we obtain

We take the reference signal as the step function and then perform simulations:

$$
r(k)= \begin{cases}2, & k \geq 50, \\ 0, & k<50 .\end{cases}
$$

Figure 1 shows the output response of the closed-loop system for system (22). Figure 2 shows the control input. It can be seen from three situations (i.e., the preview lengths of the reference signal and the exogenous disturbance are $M_{R}=2, M_{d}=2, M_{R}=5, M_{d}=2$, and no preview (namely, $\left.M_{R}=M_{d}=0\right)$ ) that the output can all track the reference signal accurately. However, both the tracking error and the input peak decrease with the increase of the preview length of the reference signal when the preview length of the exogenous disturbance is identical; namely, $M_{d}=2$; and the output of the closed-loop system can track the reference signal more quickly. Compared with no preview, in addition to the advantages mentioned above, the disturbance attenuation performance is improved by virtue of preview compensation. This is exactly how preview control achieves its goal.

In addition, from (9), the steady-state values of state variables $x(k)$ and input variables $u(k)$ are given by

$$
\begin{aligned}
& u^{*}=\lim _{k \rightarrow \infty} u(k) \approx-10.18182, \\
& x^{*}=\lim _{k \rightarrow \infty} x(k) \approx\left[\begin{array}{c}
0.14545 \\
-1.74545
\end{array}\right] .
\end{aligned}
$$

The simulations reveal that the steady-state values of $x(k)$ and $u(k)$ tend toward the theoretical values. Figure 2 shows 


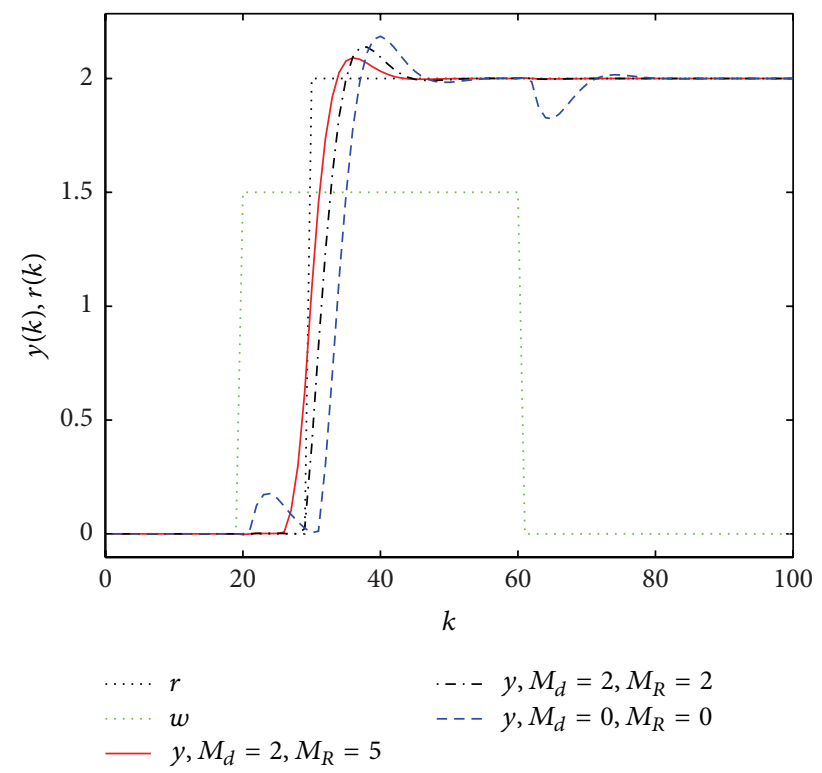

Figure 1: The output response of the closed-loop system with the same $M_{d}$ over a different $M_{R}$ and no preview.

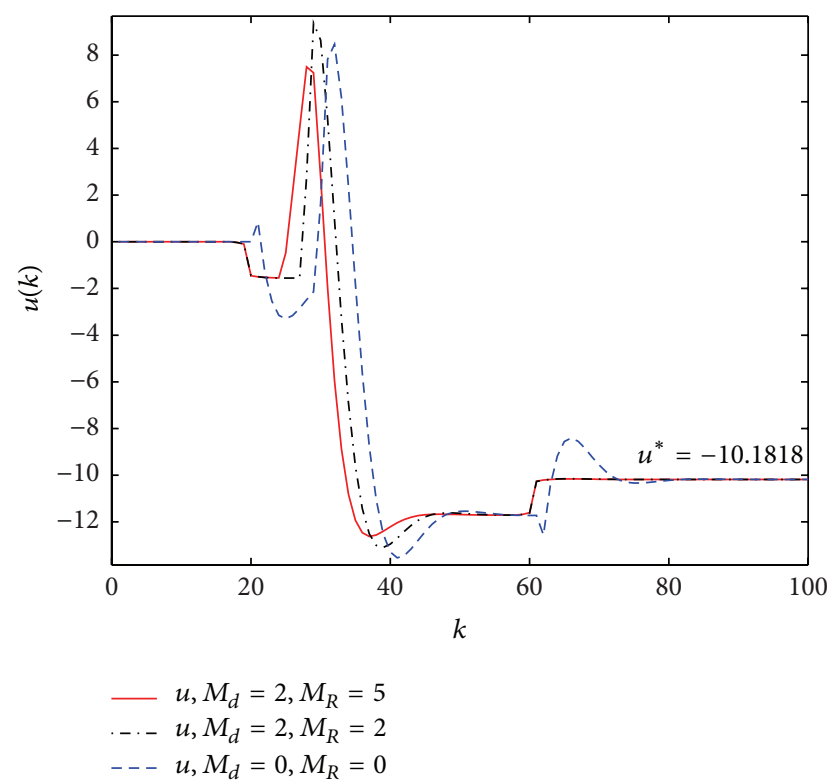

FIGURE 2: The control input of the closed-loop system with the same $M_{d}$ over a different $M_{R}$ and no preview.

the curve of the control input changing in time here as an example.

In the following, Figure 1 can be used for further analysis to illustrate the advantages of the preview controller by making use of the dynamic characteristics (rise time, peak time, settling time, etc.). The corresponding results were given out as follows:

The rise time: $k=30, k=32$, and $k=34$.

The peak time: $k=36, k=38$, and $k=40$.

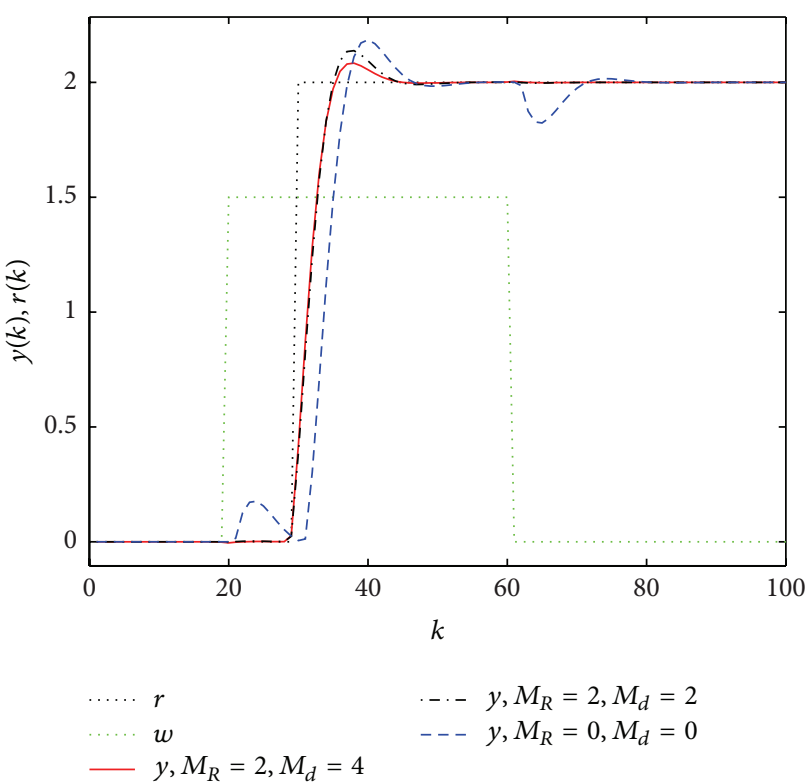

FIGURE 3: The output response of the closed-loop system with the same $M_{R}$ over a different $M_{d}$ and no preview.

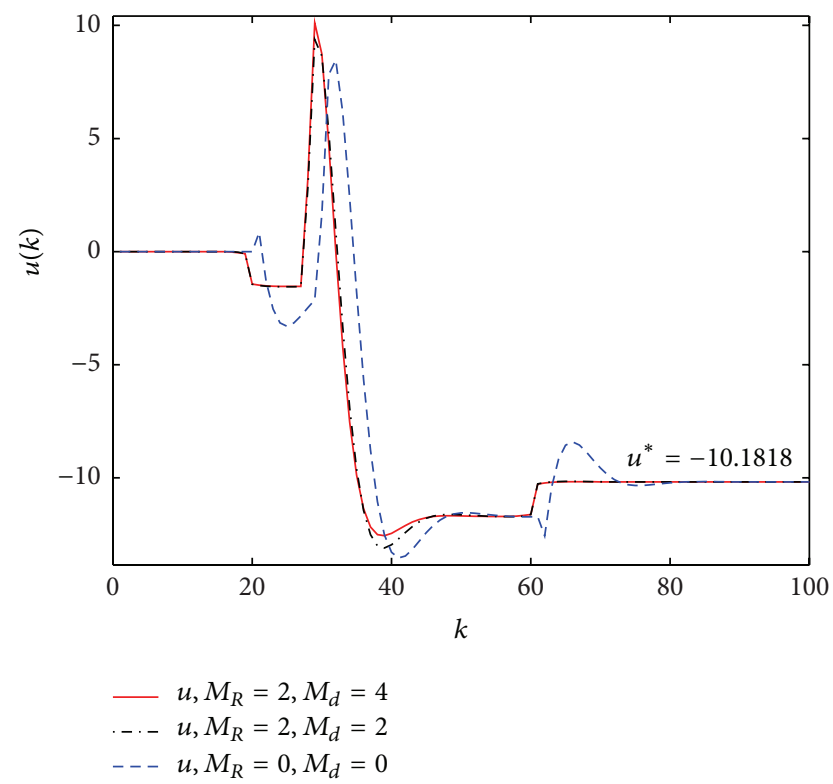

FIGURE 4: The control input of the closed-loop system with the same $M_{R}$ over a different $M_{d}$ and no preview.

The maximum overshoot: $y_{\max }=2.090914, y_{\max }=$ 2.137974 , and $y_{\max }=2.184978$.

The settling time: $k=33, k=40$, and $k=68$.

The percentage overshoot: $\sigma=4.5457 \%, \sigma=$ $6.8987 \%$, and $\sigma=9.2489 \%$.

We perform simulations for three situations; that is, the preview lengths of the reference signal and the exogenous disturbance are $M_{R}=2, M_{d}=2, M_{R}=2, M_{d}=4$, and no preview (namely, $M_{R}=M_{d}=0$ ). Figure 3 shows the output response of the closed-loop system. Figure 4 shows 
the control input. It can be seen from Figures 3 and 4 that the overshoot of the output response decreases, and the settling time can be shortened, by increasing the preview length of the disturbance signal when the preview length of the reference signal is identical; namely, $M_{R}=2$. Although, to weaken the disturbance influence over the system, the control input is increasing with the increase in preview length of the disturbance signal temporarily when the disturbance appears, the input diminishes rapidly and reaches steady state.

From the simulations for various forms of the reference signals and the disturbance signals, we find that the closedloop system can have fairly good stability, only if the reference signals or only if the disturbance signals are previewable. Now the simulation results about $M_{R}=0$ and $M_{d}=0,1,4$, respectively, will be given as an example. Figures 5 and 6 show that when the reference signal is not previewable, the overshoot of output response and the input peak decrease and the settling time can be shortened as the preview length of the disturbance signal increases.

From Figures 1, 3, and 5, we can see that the output for the system with $M_{R}=M_{d}=0$ has a larger fluctuation when the disturbance appears or disappears. However, the case in which preview action is used can effectively inhibit the fluctuation.

It follows from the simulations of periodic interference signals that the tracking error of the closed-loop system with $M_{R}=M_{d}=0$ cannot be eliminated during the survival of periodic interference signals. However, the cases in which preview action is used can eliminate the tracking error. Considering the length of this paper, the figures for these results will no longer be given out.

To design the observer, let

$$
\begin{aligned}
& L_{1}=\left[\begin{array}{c}
-5.14050 \\
2.70110
\end{array}\right], \\
& L_{2}=0.1,
\end{aligned}
$$

and we obtain

$$
M \bar{A}-\bar{L} \bar{C}=\left[\begin{array}{ccc}
-4.04132 & -4.96860 & -0.20000 \\
2.95572 & 3.64132 & 0.10000 \\
0 & 0 & -0.10000
\end{array}\right]
$$

As one can easily confirm, all the eigenvalues of matrix $M \bar{A}-$ $\bar{L} \bar{C}$ lie within the unit circle; that is, $M \bar{A}-\bar{L} \bar{C}$ is stable. Therefore, we can reconstruct the state of system (1). When the preview lengths are identical, namely, $M_{R}=8$ and $M_{d}=$ 7 , Figure 7 shows the output response of the closed loop with the extended functional observer (42) and without the observer.

Let the initial conditions be $x(0)=\left[\begin{array}{l}0 \\ 0\end{array}\right]$ and $\eta(0)=\left[\begin{array}{c}0.7 \\ -0.5 \\ 1\end{array}\right]$. From Figure 7, we can see that the oscillation caused by the deviation of initial value decays rapidly in the reconstruction process of the system state using observer (42) by choosing appropriately $L_{1}$ and $L_{2}$. And through verification, the estimation error is less than $10^{-3}$ after $k=12$ and is not affected by the disturbance (namely, (61)). The observer can give a fast

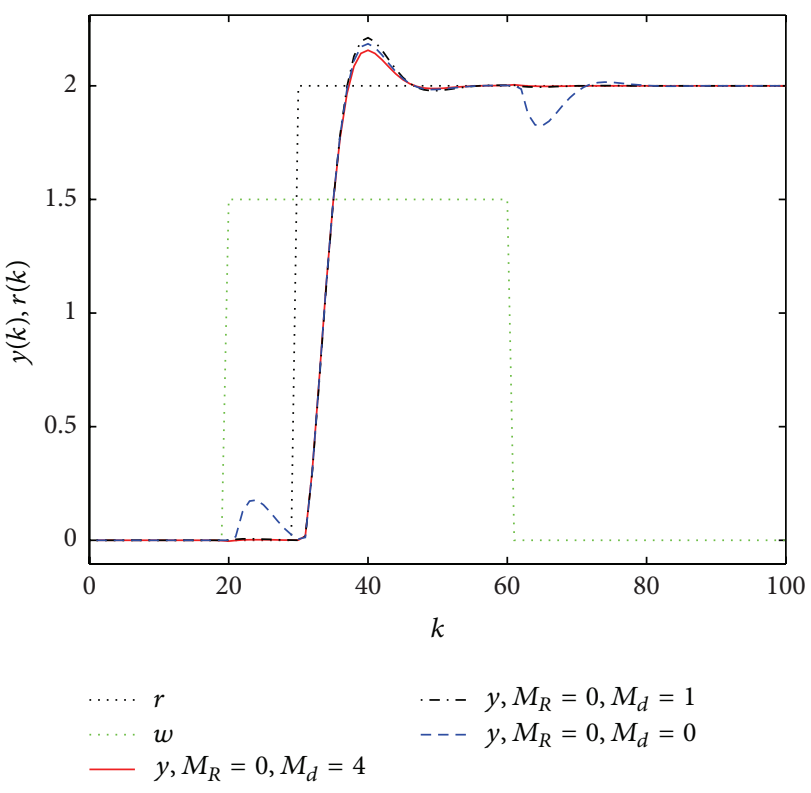

FIGURE 5: Output response of the closed-loop system with $M_{R}=0$ over a different $M_{d}$.

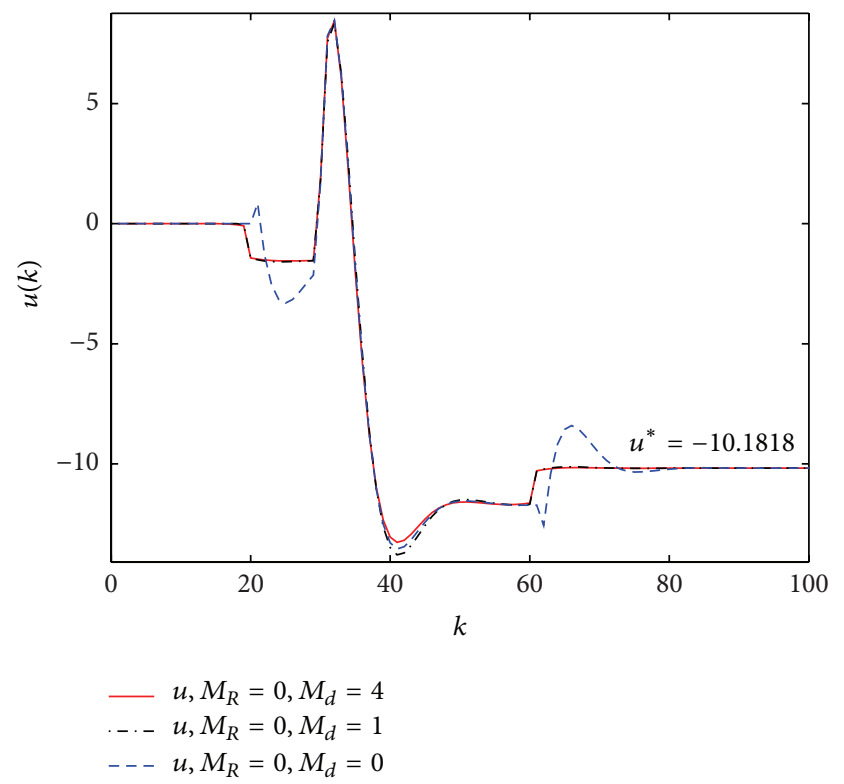

FIGURE 6: The control input of the closed-loop system with $M_{R}=0$ over a different $M_{d}$.

and accurate estimation of the state $x(k)$ and restrain the disturbance effectively. The effect of control with observer (42) is ideal.

\section{Conclusion}

In this paper, the preview control problem based on LMI is proposed for discrete-time systems. First, we adopt the method in [16] to derive an augmented system with previewable reference signal and disturbance signal. Then, by 


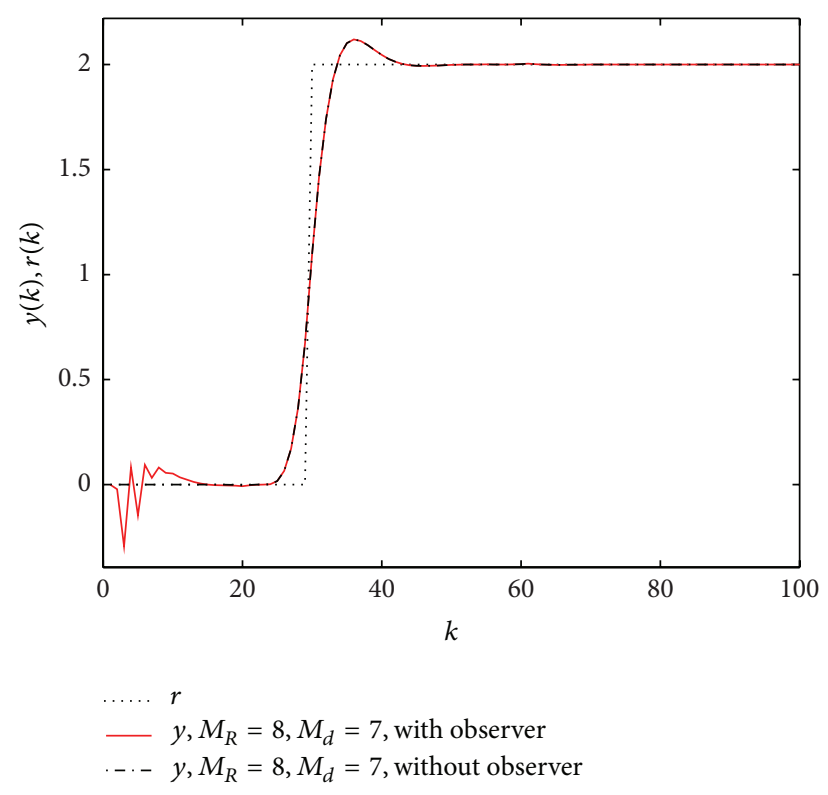

FIGURE 7: Output response of the closed-loop system with and without the observer.

introducing state feedback and applying Lyapunov stability theory, the preview controller existence condition and its design method are presented. Finally, the state feedback gain matrix can be obtained by solving an LMI problem. In addition, an extended functional observer is proposed which can speed up the convergence rate for estimation error and has the ability of anti-interference. The method used to study preview control by LMI in this paper can be extended completely into uncertain systems for preview control. The numerical simulation example also illustrates the effectiveness of the controller in this paper.

\section{Conflict of Interests}

The authors declare that there is no conflict of interests regarding the publication of this paper.

\section{Acknowledgment}

This work is supported by the National Natural Science Foundation of China (no. 61174209).

\section{References}

[1] C. Gohrle, A. Schindler, A. Wagner, and O. Sawodny, "Design and vehicle implementation of preview active suspension controllers," IEEE Transactions on Control Systems Technology, vol. 22, no. 3, pp. 1135-1142, 2014.

[2] P. Li, J. Lam, and K. C. Cheung, "Multi-objective control for active vehicle suspension with wheelbase preview," Journal of Sound and Vibration, vol. 333, no. 21, pp. 5269-5282, 2014.

[3] N. W. Verwaal, G. J. van der Veen, and J. W. van Wingerden, "Predictive control of an experimental wind turbine using preview wind speed measurements," Wind Energy, vol. 18, no. 3, pp. 385-398, 2015.
[4] A. Suzumura and Y. Fujimoto, "Real-time motion generation and control systems for high wheel-legged robot mobility," IEEE Transactions on Industrial Electronics, vol. 61, no. 7, pp. 36483659, 2014.

[5] H. P. Liu and F. C. Liao, "Design of an optimal preview controller for multirate systems with general previewable signal," Pure and Applied Mathematics, vol. 24, no. 4, pp. 634-642, 2008.

[6] Y.-J. Xu and F.-C. Liao, "Preview control for a class of timevarying discrete systems with input time-delay," Control and Decision, vol. 28, no. 3, pp. 466-470, 2013.

[7] M. J. Cao and F. C. Liao, "Design of an optimal preview controller for linear discrete-time descriptor systems with state delay," International Journal of Systems Science, vol. 46, no. 5, pp. 932-943, 2015.

[8] A. Kojima, " $H_{\infty}$ controller design for preview and delayed systems," IEEE Transactions on Automatic Control, vol. 60, no. 2, pp. 404-419, 2015.

[9] R. H. Middleton, J. Chen, and J. S. Freudenberg, "Tracking sensitivity and achievable $H_{\infty}$ performance in preview control," Automatica, vol. 40, no. 8, pp. 1297-1306, 2004.

[10] G. Marro and E. Zattoni, " $\mathrm{H}_{2}$-Optimal rejection with preview in the continuous-time domain," Automatica, vol. 41, no. 5, pp. 815-821, 2005.

[11] L. Mianzo and H. Peng, "Output feedback $H_{\infty}$ preview control of an electromechanical valve actuator," IEEE Transactions on Control Systems Technology, vol. 15, no. 3, pp. 428-437, 2007.

[12] A. A. Moelja and G. Meinsma, " $H_{2}$ control of preview systems," Automatica, vol. 42, no. 6, pp. 945-952, 2006.

[13] E. Gershon and U. Shaked, " $H_{\infty}$ preview tracking control of retarded state-multiplicative stochastic systems," International Journal of Robust and Nonlinear Control, vol. 24, no. 15, pp. 21192135, 2014.

[14] H.-X. Wang and H.-S. Zhang, "Finite horizon $H_{\infty}$ preview control," Acta Automatica Sinica, vol. 36, no. 2, pp. 327-331, 2010.

[15] S. Boyd, L. El Ghaoui, E. Feron, and V. Balakrishnan, Linear Matrix Inequalities in System and Control Theory, Society for Industrial and Applied Mathematics, Philadelphia, Pa, USA, 1994.

[16] Y. Fujisaki and T. Narazaki, "Optimal preview control based on quadratic performance index," in Proceedings of the 36th IEEE Conference on Decision \& Control, pp. 3830-3835, San Diego, Calif, USA, December 1997.

[17] K. Takaba, "Robust servomechanism with preview action for polytopic uncertain systems," International Journal of Robust and Nonlinear Control, vol. 10, no. 2, pp. 101-111, 2000.

[18] F. Liao, J. L. Wang, and G.-H. Yang, "Reliable robust preview tracking control against actuator faults," Asian Journal of Control, vol. 5, no. 1, pp. 124-131, 2003.

[19] H. Oya, K. Hagino, and M. Matsuoka, "Observer-based robust tracking control with preview action for uncertain discrete-time systems," IEICE Transactions on Fundamentals of Electronics, Communications and Computer Sciences, vol. E90-A, no. 2, pp. 517-522, 2007.

[20] F. Mazenc, V. Andrieu, and M. Malisoff, "Design of continuous-discrete observers for time-varying nonlinear systems," Automatica, vol. 57, pp. 135-144, 2015.

[21] R. E. Benton Jr. and D. Smith, "Static output feedback stabilization with prescribed degree of stability," IEEE Transactions on Automatic Control, vol. 43, no. 10, pp. 1493-1496, 1998.

[22] T. Katayama, T. Ohki, T. Inoue, and T. Kato, "Design of an optimal controller for a discrete-time system subject to previewable 
demand," International Journal of Control, vol. 41, no. 3, pp. 677699, 1985.

[23] D. Z. Zheng, Linear System Theory, Tsinghua University Press, Beijing, China, 2012.

[24] T. Tsuchiya and T. Egami, Digital Preview and Predictive Control, Translated by: F. Liao, Beijing Science and Technology Press, Beijing, China, 1994.

[25] J. H. Zhang, Y. J. Lin, and P. Shi, "Output tracking control of networked control systems via delay compensation controllers," Automatica, vol. 57, pp. 85-92, 2015.

[26] Q. P. Ha, N. D. That, P. T. Nam, and H. Trinh, "Partial state estimation for linear systems with output and input time delays," ISA Transactions, vol. 53, no. 2, pp. 327-334, 2014.

[27] M. Yang, C. Dong, S. Y. Wang, and T. Chao, "Linear extended state observer based on finite-time output feedback," Acta Automatica Sinica, vol. 41, no. 1, pp. 59-66, 2015. 


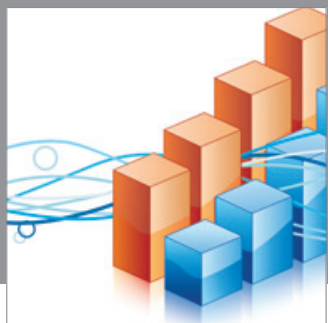

Advances in

Operations Research

mansans

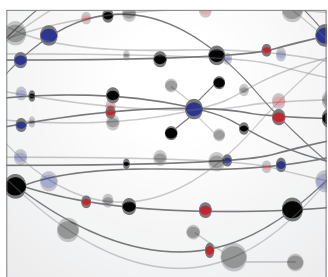

The Scientific World Journal
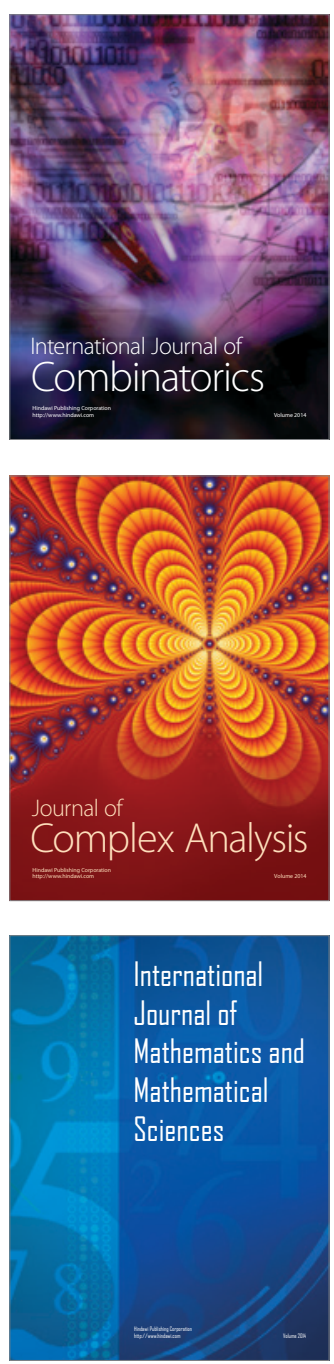
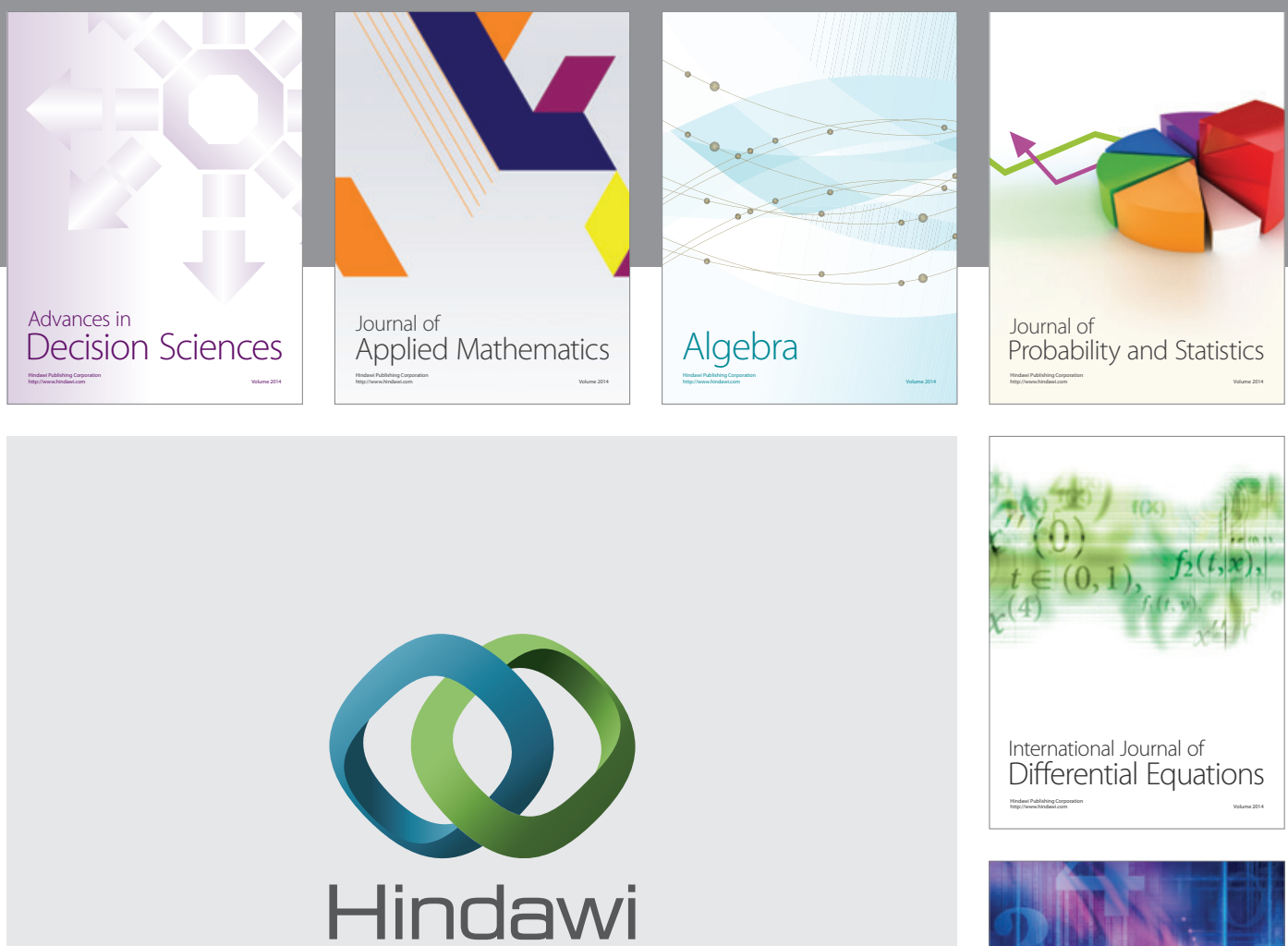

Submit your manuscripts at http://www.hindawi.com
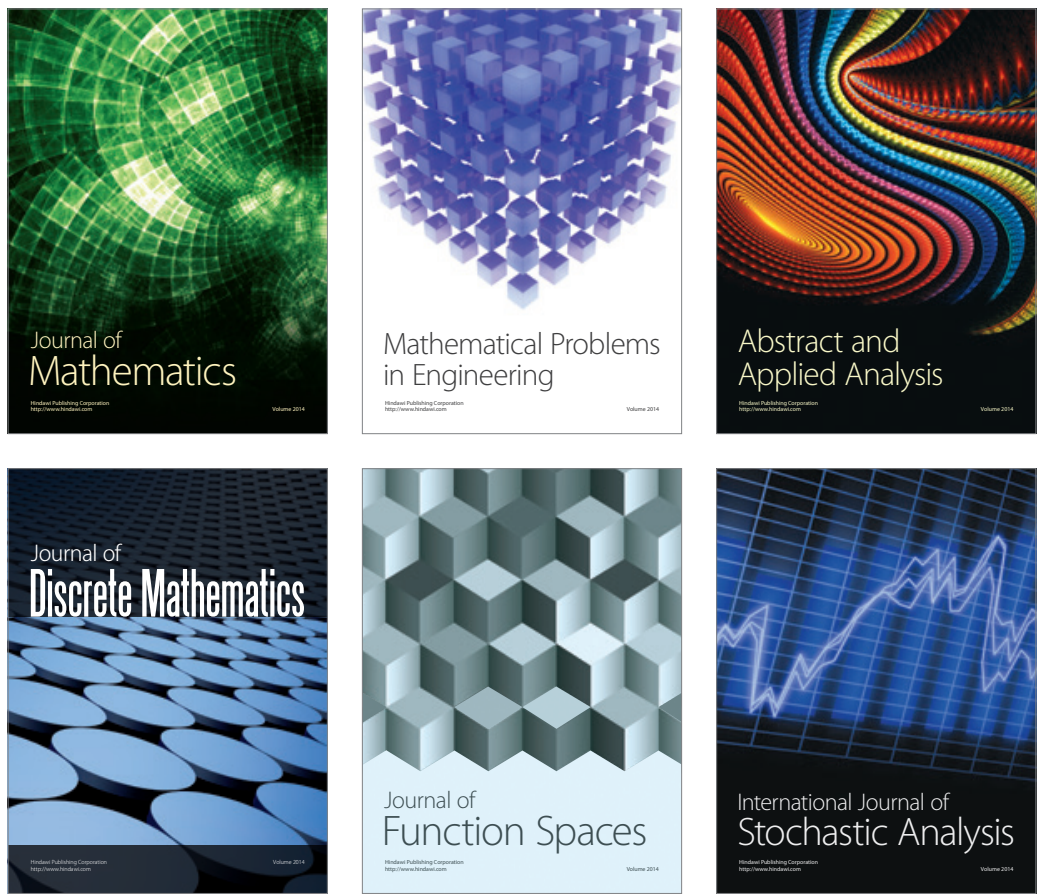

Journal of

Function Spaces

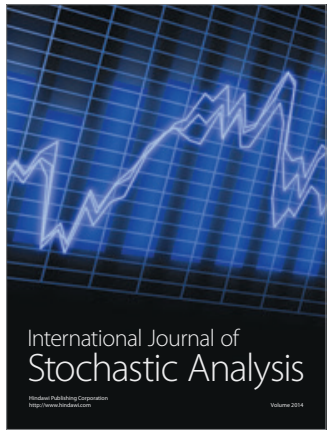

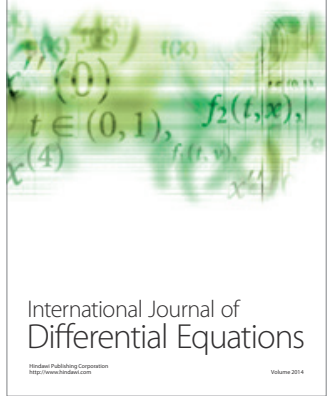
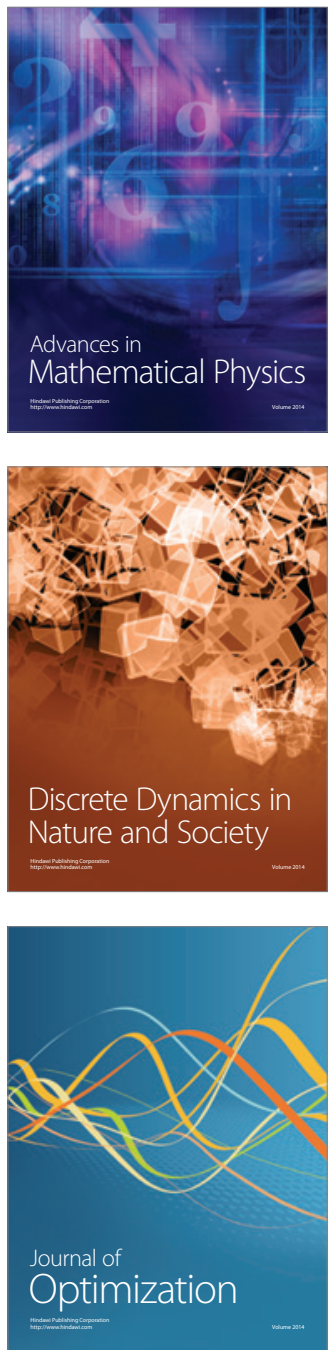